\title{
Serum endocan level and the severity of spinal cord injury
}

\author{
Ur K ${ }^{1}$, Demiroz $\mathrm{S}^{2}$, Bengu $\mathrm{AS}^{3}$, Ulucan $\mathrm{A}^{4}$, Oz Gergin $\mathrm{O}^{5}$, Kizmazoglu $\mathrm{C}^{6}$, Ozer $\mathrm{E}^{6}$ \\ Department of Neurosurgery, Bingöl State Hospital, Bingöl, Turkey. serdardemiroz@hotmail.com
}

\begin{abstract}
OBJECTIVE: To determine whether the endocan level may be related to the severity of spinal cord injury. BACKGROUND: Several biomarkers were evaluated for this purpose, but endocan has never been studied before. It is correlated to endothelial dysfunction and ischemia, which are the characteristics of spinal cord injury in most cases.

MATERIAL AND METHODS: A total of 21 male Sprague-Dawley rats weighing 300-350 g were randomly divided into three groups. In Group I, only a laminectomy was performed; in Group II, a mild SCI was performed after laminectomy; and, in Group III, a severe SCI was performed after laminectomy. At $48 \mathrm{~h}$ after the injury, after neurological assessment by Tarlov method, all animals were euthanized. A 5 cc blood sample was drawn for biochemical analysis, and spinal cord tissues were removed for histopathological examination.

RESULTS: The difference between Groups I and III was statistically significant $(p<0.05)$. There was also a moderately positive correlation between the severity of $\mathrm{SCl}$ and the endocan level $(r=0.59, \mathrm{p}<0.05)$.

CONCLUSION: The endocan level may be used as an indicator to determine prognosis after SCI (Tab. 1, Fig. 2 ,

Ref. 24). Text in PDF www.elis.sk.

KEY WORDS: Endocan, spinal cord injury, prognosis.
\end{abstract}

\section{Introduction}

Traumatic spinal cord injury (SCI) represents a delicate situation for both the patient and the physician. It may result in the loss of motor, sensory, and autonomic functions below the level of injury, which is devastating for the patient. The damage can result in a spectrum of outcomes from a complete recovery to partial or complete paraplegia, tetraplegia, or death. It was reported that $30.1 \%$ of patients had incomplete tetraplegia, $25.6 \%$ had complete paraplegia, $20.4 \%$ had complete tetraplegia, and $18.4 \%$ had incomplete paraplegia, and that the mortality rate was $17 \%$ after trauma involving SCI $(1,2)$. The level and the severity of the injury are the major determinants of the functional results. However, there is a limitation in determining the prognosis, especially in incomplete injuries.

Biomarkers may be valuable tools for assessing the severity of SCI and determining the prognosis. Several biomarkers were evaluated for this purpose, such as: neuron-specific enolase (NSE),

\footnotetext{
${ }^{1}$ Department of Neurosurgery, Bingöl State Hospital, Bingöl, Turkey, ${ }^{2}$ Department of Orthopaedics and Traumatology, Bingöl State Hospital, Bingöl, Turkey, ${ }^{3}$ Department of Medical Services and Techniques, Vocational School of Health Services, Bingol University, Bingöl, Turkey, ${ }^{4}$ Department of Veterinary Pathology, Vocational School of Health Services, Bingöl University, Bingöl, Turkey, ${ }^{5}$ Department of Anesthesiology and Reanimation, Kayseri Education and Research Hospital, Kayseri, Turkey, and ${ }^{6}$ Department of Neurosurgery, Eylül University, Izmir, Turkey

Address for correspondence: S. Demiroz, MD, Department of Orthopaedics and Traumatology, Bingöl State Hospital, Saray street, 12000 Bingöl, Turkey.

Phone: +90.505.3386490, Fax: +90.426 .2131043$
}

S100 beta (S100b) protein, glial fibrillary acidic protein (GFAP), and others. However, currently, there is no confirmed biomarker.

Endocan is a novel molecule produced by endothelial cells. It is a proteoglycan molecule that circulates freely in blood (3). Its overexpression has been shown in several disorders, such as sepsis and inflammatory diseases (4-6), and correlated to endothelial dysfunction and ischemia, which had a close relationship with SCI. The aim of this study, then, was to investigate whether the endocan level was related to the severity of SCI and whether it might be used as a prognostic biomarker.

\section{Materials and methods}

The experimental protocol was approved by the local ethics committee. A total of 21 male Sprague-Dawley rats weighing 300-350 g were included in this study and randomly divided into three groups. In Group I, only a laminectomy was performed (Sham); in Group II, a mild SCI was performed after laminectomy; and, in Group III, a severe SCI was performed after laminectomy.

All rats were kept under standard conditions at $20-22{ }^{\circ} \mathrm{C}$, at appropriate humidity under a 12 -hour light-dark cycle. They were allowed to freely access water and food. Anesthesia was induced by intraperitoneal injection of $10 \mathrm{mg} / \mathrm{kg}$ of xylazine (Alfazyne, Egevet, Izmir, Turkey) and $50 \mathrm{mg} / \mathrm{kg}$ of ketamine (Ketalar, Parke-Davis, Eczacibasi, Turkey). The rats were placed in the prone position, and their backs were shaved and cleaned with 10 $\%$ polyvidone iodine (Bathicon; Adeka, Samsun, Turkey). A midline skin incision was made between $\mathrm{T} 7$ and T12, paravertebral muscles were dissected, and a total laminectomy was performed under the microscope at the T10 level. After laminectomy, the 


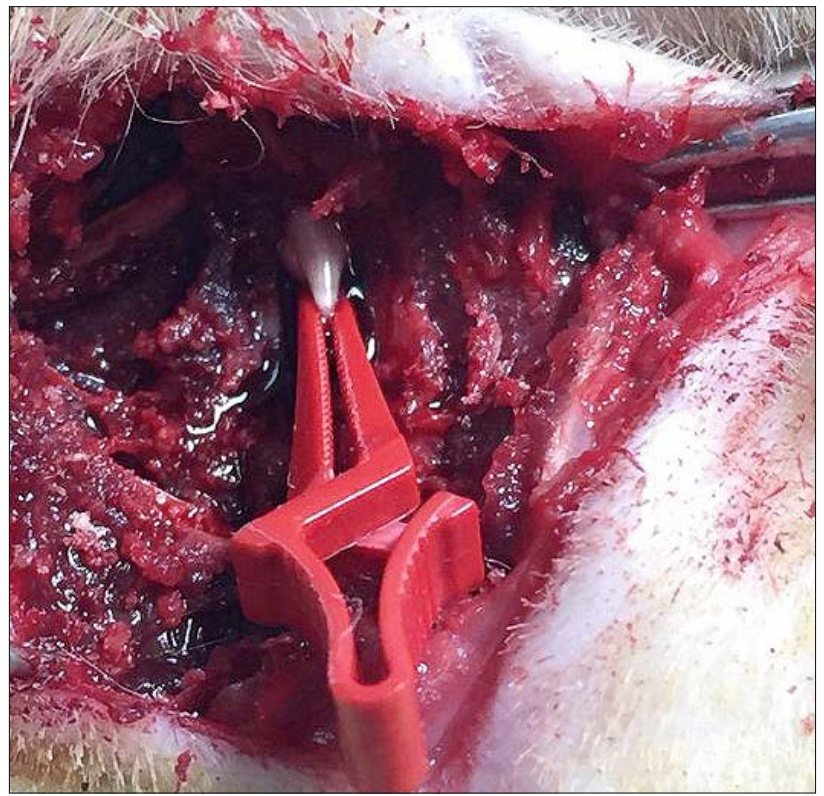

Fig. 1. Compression of the spinal cord with a clamp

surgical wound was closed without SCI in Group I. After the same procedure for approach to the spinal cord, a vascular clip (Vascu-Statt ${ }^{2}$; Scanlan, St.Paul,MN) with $20 \mathrm{~g}$ closing force was applied to the T10 level of the spinal cord in Group II, and then the wound was closed. A vascular clip (Vascu-Statt $\AA$; Scanlan, St. Paul, MN) with $40 \mathrm{~g}$ closing force was applied to the same level of the spinal cord in Group III, and then the wound was closed. We compressed the spinal cord for one min, which was sufficient to cause mild to severe damage (7) (Fig. 1). At $48 \mathrm{~h}$ after the injury, the neurological status of all animals was evaluated according to the Tarlov method (8). All animals were allowed to move freely, and an observer assessed the following: $0=$ paraplegic (absence of muscle tonus and contraction), 1 = poor motor function, $2=$ motion present, but not able to stand, $3=$ able to stand and walk, and 4 = full recovery. After neurological assessment, all animals were euthanized by decapitation under a deep anesthesia and necropsied according to the Virchow technique. A 5 cc blood sample was drawn for biochemical analysis, and spinal cord tissues were removed for histopathological examination. Blood samples were centrifuged at 1,500 rpm for $15 \mathrm{~min}$ to obtain serum, and serum samples were stored at $-80^{\circ} \mathrm{C}$ before analysis of the endocan levels by enzyme-linked immunosorbent assay (ELISA). The spinal cord tissues were fixed in $10 \%$ neutral-buffered formalin for 48 $\mathrm{h}$ immediately after removal. The tissue samples were processed following the routine protocol; they were dehydrated in ascending grades of ethanol $(50 \%, 70 \%, 96 \%$, and $100 \%)$, cleared in xylene, and then embedded in paraffin. Paraffin-embedded tissue samples were sectioned at $5 \mu \mathrm{m}$ thickness by a rotary microtome (Leica RM2125; Leica, Nussloch, Germany). The slides were stained with hematoxylin and eosin (H\&E) for histological examination (Lilli, 2008). Finally, the slides were examined and photographed using a light microscope with an imaging system (DM2500/DFC295; Leica, Nussloch, Germany). The pathological findings were evaluated and the prevalence of inflammatory reaction (PIR), which indicated cellular damage, was scored according to the percentage of glial cell proliferation in the region of the tissue damage with $10 \mathrm{x}$ objective by a pathologist. If there was no proliferation, it was scored as 0 ; if there was proliferation, it was scored as followed: $<1 \%=1 ; 1-9 \%=2 ; 10-32 \%=3 ; 33-65 \%=4$, and $>65 \%=5(9)$.

\section{Statistical analysis}

All data were analyzed using the SPSS 10.0 software package (SPSS Inc., Chicago, IL, USA). The Mann-Whitney $U$ test was used to compare the descriptive statistical measurements (mean, standard deviation $(S D)$, median, frequency, ratio, minimum, and maximum) and the two groups of parameters that did not show a normal distribution in the comparison of the quantitative data. One way analysis of variance (ANOVA) was applied to assess the differences among the groups. Pearson's correlation analysis was performed to determine the significance of the correlation. Significance was evaluated at $\mathrm{p}<0.05$.

\section{Results}

Neurological examinations were assessed $48 \mathrm{~h}$ after the surgery. All of the rats were capable of walking, and the differences among the Tarlov scores in the three groups were not statistically significant $(p>0.05)$ (Tab. 1).
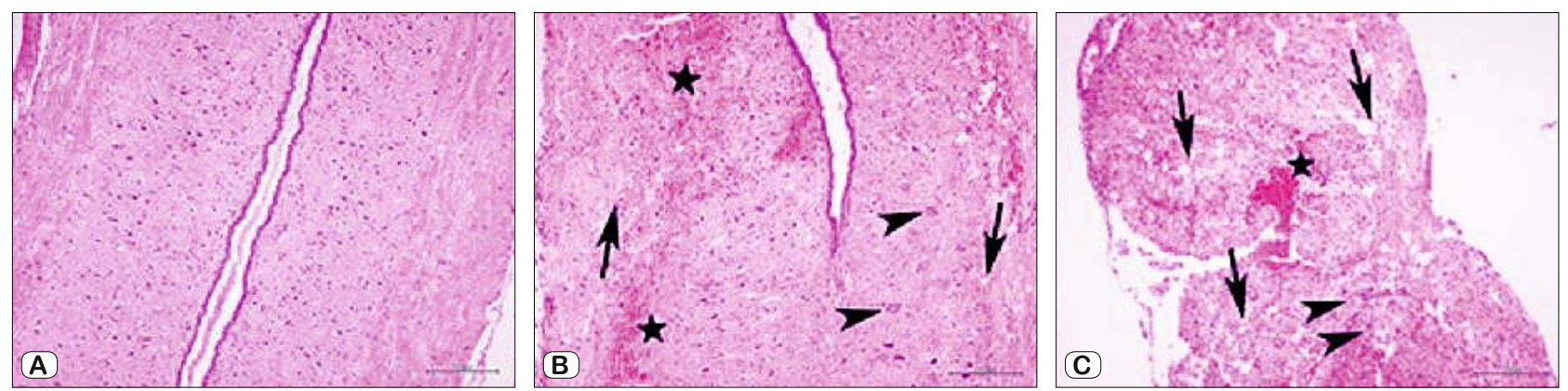

Fig. 2. Histopathology of the spinal cord tissues; magnification x10, H\&E staining. (A) Group 1: normal histological structure of the medulla spinalis; (B) Group 2: degeneration of the neurons (arrow heads), mild congestion (stars), and spongious appearances (arrows) in the white matter; (C) Group 3: severe neuronal loss and proliferation of the glial cells (arrow heads), diffuse spongious appearances (arrows) in the white matter, and diffuse hemorrhage (star). 


\section{8-301}

In the evaluated spinal cord tissues, there were no significant histopathological differences in Group I, and the spinal cord tissues showed normal histological structures (Fig. 2A). In Group II, there was mild perineural vacuolation of the neurons of the motor nucleus, degeneration of some of the neurons with a pyknotic nucleus, and neuronal shrinkage and proliferation of the glial cells around the central canal and the posterior horn in the gray matter. Mild spongious appearance (degeneration of myelin sheath) was observed in the white matter of the medulla spinalis and mild congestion of blood vessels in the pia mater Figure 2B). In Group III, common lesions in the spinal cord tissues were the following: severe perineural vacuolation of the neurons of the motor nucleus, degeneration and necrosis of most of the neurons with pyknotic nucleus, neuronal shrinkage, loss of the neurons and diffuse hemorrhage with the loss of gray matter, and proliferation of the glial cells around the central canal and the posterior horn. Severe spongious appearance (axonal demyelination) was observed in the white matter of the medulla spinalis, and there was mild congestion of blood vessels in the pia mater (Fig. 2C). Mean IRSs were $0,2.75$, and 4.5 for Groups I, II, and III, respectively. Differences between IRSs were statistically significant among the three groups $(p<0.05)$ (Tab. 1). These histopathological results indicated our achievement to produce spinal cord damage at different severities.

The mean endocan level was $6.11 \mathrm{pg} / \mathrm{ml}$ in Group I, 9.23 $\mathrm{pg} / \mathrm{ml}$ in Group II, and 10.73 in Group III. Differences between Groups I and II, and Groups II and III were not statistically significant ( $p>0.05)$, but the difference between Groups I and III was statistically significant $(\mathrm{p}<0.05)$ (Tab. 1$)$. There was also a moderate positive correlation between the severity of SCI and endocan level $(\mathrm{r}=0.59, \mathrm{p}<0.05)$.

\section{Discussion}

We found that there was a statistically significant difference between Groups I and III regarding endocan levels, although the rats had similar neurological examination scores. Furthermore, there was also a moderate positive correlation between the severity of SCI and endocan levels.

The most common mechanism of SCI in humans is a combination of acute impaction and persistent compression (10). First, the vertebral fragments are propelled into the spinal canal and lead to contusion, resulting in a compression of the cord. This first effect is called the primary injury. The secondary injury state starts after the primary injury. It is characterized by ischemic dysfunction, in-

Tab. 1. Tarlov and Inflammatory reaction scores and endocan level of three groups.

\begin{tabular}{lccc}
\hline & $\begin{array}{c}\text { Group I } \\
(\mathrm{n}=7)\end{array}$ & $\begin{array}{c}\text { Group II } \\
(\mathrm{n}=7)\end{array}$ & $\begin{array}{c}\text { Group III } \\
(\mathrm{n}=7)\end{array}$ \\
\hline Tarlov Score & $4.00 \pm 0.00$ & $3.43 \pm 0.20$ & $3.57 \pm 0.20$ \\
Inflammatory Reaction Score & - & $2.71 \pm 0.28$ & $4.43 \pm 0.20$ \\
Endocan Level $(\mathrm{pg} / \mathrm{ml})$ & $6.11 \pm 0.47$ & $9.23 \pm 1.64 *$ & $10.73 \pm 0.77 * *$ \\
\hline $\mathrm{p}>0.05$ when the three groups were compared according to the Tarlov score with \\
each other, $\mathrm{p}<0.05$ when the three groups were compared according to the inflam- \\
matory reaction score with each other, * $\mathrm{p}>0.05$ vs group 1, ** $\mathrm{p}<0.05$ vs group 1, \\
$\mathrm{p}>0.05$ when the group 2 and 3 compared
\end{tabular}

flammation, oxidative stress, and neuronal injury, and this period may last for weeks. Because this is the method, which most likely resembles the real SCI mechanism in humans, we used vascular clips for compression, which have different closure pressures, on a vertical plane to create SCI of different severities. In this method, at the beginning of the closure, a contusion is caused by the clip blades, which leads to disruption of the spinal cord and hemorrhage, and, after completely closing the blades, the compression phase starts, which leads to ischemia (7).

Because of the absence of an effective therapy, the prognosis of SCI patients primarily depends on the severity of injury. Although it is obvious that complete injuries have a poor prognosis, it is not possible to estimate the prognosis for an incomplete injury. The neurological examination, MRI assessment, and electrophysiological examination may all offer insights into the severity of the SCI and prognosis, but they all have limitations. For example, neurological examination is not suitable for the patients, who have complicated extremity fractures or who are not cooperative. Spinal shock may also develop after the injury, which may result in the absence of reflexes. On the other hand, if the neurological examination is not possible, MRI may provide some prognostic information, but, if the patient has an uncomplicated injury with a stable neurological condition, MRI does not provide additional prognostic information. Another method that may be used as a prognostic factor, electrophysiological examination, is not indicated in the evaluation of cooperative patients with SCI. Because of these limitations, Middendorp et al. emphasized the importance of and the need to find possible biomarkers for SCI (11).

Because they are objectively quantifiable, easy to apply, and able to provide unbiased results, it is logical that the biomarkers may be valuable tools for assessing the severity of SCI. Ma et al. presented the increase in serum concentration of S100 beta protein in rats after SCI. S100 beta protein is mostly found in glial cells, and there are studies indicating its increase after traumatic brain injury. Therefore, it was reasonable to hope that S100 beta protein increases in blood. As a result, they suggested that the concentration of $\mathrm{S} 100$ beta protein in serum might be used as an early diagnostic tool after SCI (12). Loy et al also noted the elevation of NSE and S100 beta protein levels after an acute experimental SCI, and they suggested that these two neural tissue-derived tools may be used to assess the damage to the spinal cord (13). Cao et al also suggested the elevations of NSE and S100 beta protein after SCI, not only in serum, but also in the cerebrospinal fluid (CSF), and emphasized that they could be used as biomarkers for acute SCI (14). Guez et al found significantly increased concentrations of both GFAP and neurofilament (NFL) in the CSF in six cases with spinal cord damage (15). Kwon et al. obtained CSF and blood samples from 27 patients with complete or incomplete SCI. They measured cytokines by using the cytokine array system and standard ELISA techniques. When they compared the neurological recovery with the level of these cytokines, they found that IL-6, IL-8, MCP-1, tau, S100b, and GFAP were elevated in parallel to the severity of the injury (16). Pouw et al. reviewed 18 studies concerning biomarkers related to SCI. They found that most of the studies were focused on S100 beta and NSE levels and that, 
although the results were promising, there are currently no validated biomarkers (17).

Next, we considered whether a novel endocan molecule might be a biomarker for the severity of SCI. This is a proteoglycan molecule, which is specific to the endothelial cells. Several studies showed that the endocan molecule was overexpressed in some cancers (18-21). There was a correlation determined between the involvement of endocan and angiogenesis. Furthermore, it was also indicated that endocan might play a role in endothelial dysfunction (22-24). Sepsis and inflammation are also associated with endothelial dysfunction, as well as vasodilation, edema, coagulopathy, ischemia, and organ failure. When considering the relationship between endocan and endothelial dysfunction, ischemia, and inflammation, we thought that it might be a biomarker related to SCI. When considering the pathophysiology of the spinal damage, secondary injury is also characterized by ischemia and inflammation.

On the other hand, our study had several limitations: there was a relatively short follow-up period, samples were not taken from CSF, and blood samples were taken only during sacrifice, rather than at different times after the trauma.

In conclusion, our study supports that the endocan level increases in parallel to the severity of SCI in incomplete injuries; therefore, it may be used as a prognostic biomarker in SCI for determining the severity of the injury. We believe that our results are an important contribution to literature considering the difficulty and limitation in predicting prognosis in SCIs.

\section{Learning points}

When considering the pathophysiology of SCI, endocan might be a biomarker for severity and prognosis.

\section{References}

1. National Spinal Cord Injury Statistical Center (SCISC). The 2008 annual statistical report for the spinal cord injury model systems. Available at: https://www.nscisc.uab.edu/public content/pdf/2008\%20NSCISC $\% 20$ Annual\%20Statistical\%20Report\%20-\% 20Complete\%20Public\%20Version.pdf.

2. Pirouzmand F. Epidemiological trends of spine and spinal cord injuries in the largest Canadian adult trauma center from 1986 to 2006. J Neurosurg Spine 2010; 12: 131-140.

3. Lassalle P, Molet S, Janin A et al. ESM-1 is a novel human endothelial cell-specific molecule expressed in lung and regulated by cytokines. J Biol Chem 1996; 271: 20458-20464.

4. Scherpereel A, Depontieu F, Grigoriu B et al. Endocan, a new endothelial marker in human sepsis. Crit Care Med 2006; 34: 532-537.

5. Balta I, Balta S, Demirkol S et al. Elevated serum levels of endocan in patients with psoriasis vulgaris: correlations with cardiovascular risk and activity of disease. Brit J Dermatol 2013; 169: 1066-1070.

6. Balta I, Balta S, Koryurek OM et al. Serum endocan levels as a marker of disease activity in patients with Behcet disease. J Amer Acad Dermatol 2014; 70: 291-296.

7. Marques SA, Garcez VF, Del Bel EA, Martinez AM. A simple, inexpensive and easily reproducible model of spinal cord injury in mice: Morphological and functional assessment. J Neurosci Methods 2009; 177: 183-193.
8. Kerasidis H, Wrathall JR, Gale K. Behavioral assessment of functional deficit in rats with contusive spinal cord injury. J Neurosci Methods 1987; 20: 167-79.

9. Vermeirsch H, Simoens P, Coryn M, Van den Broeck W. Immunolocalization of androgen receptors in the canine ovary and their relation to sex steroid hormone concentrations. Reproduction 2001; 122: 711-721.

10. De Girolami U, Frosch MP, Tator CH. Regional neuropathology diseases of the spinal cord and vertebral column. In: Graham DI, Lantos PL (Eds). Greenfield's Neuropathology. London: Arnold, 2002, 1063-1101.

11. van Middendorp JJ, Goss B, Urquhart S, Atresh S, Williams RP, Schuetz M. Diagnosis and Prognosis of Traumatic Spinal Cord Injury. Global Spine J 2011; 1: 1-8.

12. Ma J, Novikov LN, Karlsson K, Kellerth JO, Wiberg M. Plexus avulsion and spinal cord injury increase the serum concentration of S-100 protein: an experimental study in rats. Scand J Plast Reconstr Surg Hand Surg 2001; 35: 355-359.

13. Loy DN, Sroufe AE, Pelt JL et al. Serum biomarkers for experimental acute spinal cord injury: Rapid elevation of neuron-specific enolase and S-100 beta. Neurosurgery 2005; 56: 391-397.

14. Cao F, Yang XF, Liu WG et al. Elevation of neuron-specific enolase and $\mathrm{S} 100$ beta protein level in experimental acute spinal cord injury. J Clin Neurosci 2008; 15: 541-544.

15. Guéz M, Hildingsson C, Rosengren L, Karlsson K, Toolanen G. Nervous tissue damage markers in cerebrospinal fluid after cervical spine injuries and whiplash trauma. J Neurotrauma 2003; 20: 853-858.

16. Kwon BK, Stammers AM, Belanger LM et al. Cerebrospinal fluid inflammatory cytokines and biomarkers of injury severity in acute human spinal cord injury. J Neurotrauma 2010; 27: 669-682.

17. Pouw MH, Hosman AJF, van Middendorp JJ, Verbeek MM, Vos PE, van de Meent H. Biomarkers in spinal cord injury. Spinal Cord 2009; 47: 519-525.

18. Roudnicky F, Poyet C, Wild P et al. Endocan is upregulated on tumor vessels in invasive bladder cancer where it mediates VEGF-A-induced angiogenesis. Cancer Res 2013; 73: 1097-1106.

19. Chen LY, Liu X, Wang SL, Qin CY. Over-expression of the Endocan gene in endothelial cells from hepatocellular carcinoma is associated with angiogenesis and tumour invasion. J Internat Med Res 2010; 38: 498-510.

20. Grigoriu BD, Depontieu F, Scherpereel A et al. Endocan expression and relationship with survival in human non-small cell lung cancer. Clin Cancer Res 2006; 12: 4575-4582.

21. Leroy X, Aubert S, Zini L et al. Vascular endocan (ESM-1) is markedly overexpressed in clear cell renal cell carcinoma. Histopathology 2010; 56: $180-187$.

22. Yilmaz MI, Siriopol D, Saglam M et al. Plasma endocan levels associate with inflammation, vascular abnormalities, cardiovascular events, and survival in chronic kidney disease. Kidney Internat 2014; 86: 1213-1220.

23. Balta S, Mikhailidis DP, Demirkol S, Ozturk C, Celik T, Iyisoy A. Endocan: a novel inflammatory indicator in cardiovascular disease? Atherosclerosis 2015; 243: 339-343.

24. Xiong C, Zhao ZW, Chen ZY et al. Elevated human endothelial cellspecific molecule-1 level and its association with coronary artery disease in patients with hypertension. J Invest Med 2015; 63: 867-870.

Received January 11, 2018. Accepted February 27, 2018. 